\title{
Essais
}

Revue interdisciplinaire d'Humanités

1 | 2012

Varia

\section{Entretien avec Carlos Fuentes}

Estelle Patoyt et Andrea Cabezas Vargas

Traducteur : Anne-Laure Rebreyend

\section{CpenEdition}

Journals

Édition électronique

URL : https://journals.openedition.org/essais/10630

DOI : $10.4000 /$ essais. 10630

ISSN : 2276-0970

Éditeur

École doctorale Montaigne Humanités

Édition imprimée

Date de publication : 15 mai 2012

Pagination : 18-25

ISSN : 2417-4211

Référence électronique

Estelle Patoyt et Andrea Cabezas Vargas, «Entretien avec Carlos Fuentes », Essais [En ligne], 1 | 2012, mis en ligne le 31 janvier 2022, consulté le 02 février 2022. URL : http://journals.openedition.org/ essais/10630; DOI : https://doi.org/10.4000/essais.10630 


\section{Mené par Estelle Patoyt et Andrea Cabezas Vargas}

Université Michel de Montaigne Bordeaux 3, EA 4426 MICA et Universidad de Costa Rica, Doctorado en estudios de Sociedad y Cultura.

Mail : andrea.cabezas-vargas@u-bordeaux3.fr,

\section{Traduction Anne-Laure Rebreyend}

Université Michel de Montaigne Bordeaux 3, EA 3656 AMERIBER Mail : al.rebreyend@gmail.com

\section{NDLR}

Entretien réalisé à l'occasion de la venue de Carlos Fuentes à l'Université Michel de Montaigne Bordeaux 3, où il a reçu les insignes de Docteur Honoris Causa le 20 octobre 2011. 


\section{Entretien avec Carlos}

Fuentes

I - L'interculturalité et la vision de 1'autre dans l'œuvre de Carlos Fuentes

Dans l'œuvre de Carlos Fuentes, la vision de l'autre est un élément récurrent qui subit différentes métamorphoses, du nationalisme à la xénophobie, de l'acculturation aux processus de multiculturalité et interculturalité. Cette vision de l'autre est-elle une fascination omniprésente dans votre œuvre?

En Amérique Latine, nous passons beaucoup de temps à nous demander qui nous sommes. Le discours de l'identité nous a pris des années et nous a épuisés, jusqu'à parvenir à conclure que oui, nous avions une identité, que je suis mexicain, que García Márquez est colombien, Vargas Llosa Péruvien; ce n'est plus un problème.

Le problème est d'avoir une identité, et les formes de la diversité, car la vie démocratique va dépendre des formes de la diversité politique, morale, sexuelle, religieuse... ; nous sommes en train de passer d'une identité acquise à une diversité encore à conquérir.
Je ne sais si cela répond à votre question, parce qu'elle place mon œuvre au cour de tout cela. Mon œuvre tout à la fois affirme une identité - celle du Mexique, de l'Amérique Latine - et part en quête de l'autre, de l'altérité, comme on disait autrefois, en faisant de la diversité l'objectif de l'Amérique Latine actuelle.

Du fait que vous avez vécu personnellement dans des contextes de bilinguisme et de multiculturalité, pourrions-nous affirmer que votre littérature est une littérature "des deux côtés ", des deux frontières, des deux mondes?

J'ai grandi aux États-Unis, au Chili et en Argentine, mais j'ai conservé mon identité pour bien des raisons. D'abord du fait de la situation politique, la Révolution mexicaine, durant la présidence de Cárdenas qui a exproprié le pétrole, a mené la réforme agraire, a permis la syndicalisation des ouvriers, toute une série d'actes révolutionnaires. Et mon père, qui était conseiller légal de l'ambassade à Washington, me disait lorsque j'étais petit : "C'est cela le Mexique, tu es mexicain, cela fait partie de ta vie». 
Aussi n'ai-je jamais perdu ni l'identité mexicaine, ni même l'accent. Je suis venu en Europe pour la première fois à 21 ans, je me suis senti très à l'aise dans les pays européens; dès lors j'ai mené une vie transcontinentale et en lien avec les États-Unis, mais sans jamais perdre l'affiliation mexicaine.

Entretenez-vous une relation particulière avec la langue anglaise? Dans votre ouvre, elle est présente de manière explicite sous forme de prières, de mots, mais aussi indirectement par la convocation (par les épigraphes, les citations, les personnages) d'auteurs comme Yeats, Ambrose Pierce, Thomas Browne, etc.

J'ai étudié aux États-Unis durant mon enfance, et j'ai toujours pensé qu'il fallait troubler la pureté de la langue. J'aurais pu être un écrivain de langue anglaise, mais j'ai songé que les Yankees avaient déjà tout pour eux, les Faulkner, les Hemingway, les Scott Fitzgerald, qu'ils n'avaient pas besoin de moi. En revanche, en Amérique Latine, avec les gens de ma génération, comme Cortázar ou García Márquez, nous nous sommes efforcés de créer une tradition littéraire nouvelle et le défi était bien plus grand en espagnol, la langue qui avait donné ni plus ni moins que Cervantes et l'inauguration du roman moderne, mais qui depuis s'était endormie. Il n'y a peut-être que deux grands romanciers espagnols au $\mathrm{XIX}^{\mathrm{e}}$ siècle : Clarín et Pérez Galdós, puis le silence avant et après le $\mathrm{XX}^{\mathrm{e}}$ siècle. Alors j'ai senti qu'en Amérique Latine il y avait beaucoup à dire. J'ai eu d'importants mentors littéraires: Carpentier, Borges, Asturias...

Notre génération, celle qu'on a appelée le "boom ", a raconté de nouveau l'histoire d'Amérique Latine, en essayant de dire ce qui avait eu lieu et n'avait jamais été dit. Des oeuvres comme Cent ans de solitude ou La Mort d'Artemio Cruz, entre autres nombreux romans, relèvent de cette entreprise. Mais ce n'est pas celui des écrivains qui abordent les problèmes de la réalité ou de l'imaginaire d'aujourd'hui : amours, divorces, scandales, vols, possessions, dépossessions, enfants, grands-parents, que sais-je encore. Ils traitent de tous les thèmes traditionnels du roman et de la réalité, les abordent comme fiction ou non, mais ils ne font plus face au défi qui était le nôtre. Et les écrivains d'Amérique Latine sont bien plus libres aujourd'hui.

Quelles sont les principales ou les véritables frontières qui opposent les cultures du Nord et celles du Sud?

Je crois qu'il y a une double invasion, dans le cas du Mexique et des États-Unis. Les États-Unis n'ont que deux voisins : le Canada et le Mexique. C'est pourquoi le lien est plus fort avec le Mexique qu'avec l'Argentine ou le Chili. Cinquante millions de personnes parlent espagnol aux États-Unis tandis qu'au Mexique on doit trouver cent personnes qui parlent anglais - j'exagère à peine. 
La présence de la langue espagnole est énorme dans les coutumes, la cuisine, les chansons, les danses, la poésie... Tout cela est déjà entré dans un monde nord-américain qui, en outre, subit l'influence du Japon, des Philippines, de l'Europe constamment, et du monde africain.

Nous faisons partie de ce "melting-pot ": nous sommes très présents dans la vie nord-américaine. Beaucoup de Mexicains ou de Latino-américains restent aux États-Unis et deviennent des citoyens de langue anglaise. Nombre d'entre eux sont domestiques à Chicago, Los Angeles ou New York, et parfois, lorsqu'ils parviennent à rentrer au Mexique, une communauté nouvelle se crée. Ainsi les États-Unis influencent-ils l'Amérique Latine et l'Amérique Latine les Etats-Unis.

Naturellement, le pouvoir nord-américain assure que l'influence nord-américaine est grande, mais voyez plutôt la force de la culture face aux réalités politiques et économiques du moment: le Mexique est un pays voisin où l'on parle espagnol, où l'on cultive une mémoire aztèque, avec des églises baroques - même les athées y sont catholiques!-, une sensibilité et une cuisine par bonheur bien différentes de celles des États-Unis. Être voisin des ÉtatsUnis n'est pas chose facile, et pourtant le Mexique a conservé une personnalité bien plus latino-américaine que nord-américaine - avec des marques d'influence bien entendu.

\section{II - Les symboles dans La fron- tière de verre et Le vieux gringo}

Dans Le Vieux Gringo, et plus particulièrement dans La Frontière de verre, surgissent nombre de références à la Guerre d'Álamo (184648), durant laquelle le Mexique a perdu $55 \%$ de son territoire du Nord. Ce fait historique est-il une blessure ouverte pour le peuple mexicain?

Non, la perte de la moitié du territoire n'est en aucune manière un conflit pour le Mexique, et nous ne disons pas stupidement "Allons reconquérir Los Angeles ou Austin, Texas", non. Le conflit vient des faits actuels, surtout de la présence de cinquante millions de travailleurs de langue espagnole aux États-Unis, en majorité des Mexicains et des Portoricains.

En premier lieu, notre problème est : quelles sont les raisons économiques qui expliquent qu'un travailleur quitte l'Afrique pour l'Europe, ou le Mexique ou le Guatemala pour les États-Unis? Son pays d'origine a besoin de lui, il faudrait qu'il puisse y trouver du travail. C'est là un problème très grave, qui est celui de l'organisation mondiale, et qui va nous dévorer si nous ne le résolvons pas.

En second lieu, c'est un problème pour les États-Unis, car ces travailleurs ne sont pas des criminels et sont pourtant parfois traités comme tels. Quel est leur statut? Quels sont leurs droits, leurs devoirs? Comment se comportent-ils dans la société nord-américaine? C’est donc un 
conflit qui relève de la politique interne du Mexique et de la relation bilatérale entre le Mexique et les ÉtatsUnis. C'est cela le problème, ce qui s'est passé il y a plus de cinquante ans n'a d'intérêt que pour la mémoire ou le sentimentalisme.

Dans La Frontière de verre, le narrateur nous parle du désir de José Francisco :

«Je voulais donner la parole à toutes les histoires que j'entendais depuis mon enfance, des histoires de famille surtout, qui étaient la richesse du monde frontalier, toutes ces histoires encore vivantes, qui refusaient de mourir, qui allaient, libres comme des fantômes de la Californie au Texas, en attendant que quelqu'un les raconte, les écrive... convaincu que les mots voleraient jusqu'à trouver leur destin, leurs lecteurs, leurs auditeurs, leurs langues... ${ }^{1}$

Attention, ça, c'est la voix du personnage. Les écrivains n'écrivent pas pour se donner la parole à eux-mêmes, - ce serait de la mauvaise littérature - mais pour prêter une voix propre aux personnages, équivoque. Un bon roman a toujours une fin ouverte. C'est le lecteur, et non l'auteur, qui donne un avenir au roman.

Je crois lire dans votre ouvre une équivalence entre le Mexique et la mort: dans Gringo viejo, le personnage, un habitant des ÉtatsUnis, fuit au Mexique, en pleine révolution, pour y mourir; dans votre nouvelle En buena compañia, le personnage finit par appartenir à ses deux tantes, qui vivent enfermées dans une vieille maison, bloquées dans un passé morbide : à la fin du récit, elles célèbrent les funérailles de leur neveu en l'enfermant de force dans un cercueil souterrain; dans Apolo y las putas, le protagoniste déclare qu'il prend conscience de sa "mexicanité ", qu'il devient mexicain au moment de mourir.

Le Mexique a la particularité de ne pas distinguer la vie et la mort. J'insiste beaucoup sur cela dans mes livres parce que je crois que c'est une spécificité toute mexicaine, que je ne trouve pas en France ou aux États-Unis, où la culture occidentale a créé une dichotomie entre la vie, d'un côté, et la mort, de l'autre. Au Mexique nous savons que la mort fait partie de la vie, tout simplement; nous le croyons profondément, nous le vivons, avec le jour des morts, ses fleurs et ses prières et ses conversations avec les morts. C'est un fait culturel d'identité.

\section{III - Carlos Fuentes et la littérature}

Comment se manifeste la dualité entre la littérature et la lecture dans vos récits?

Il y a plus de lecteurs que d'écrivains, et plus de réel dans la réalité que dans la littérature; on aura beau écrire, on n'embrassera qu'une petite parcelle de réalité. Pourtant, je ne 
peux pas comprendre le monde sans Cervantes, sans Don Quichotte, mais avant 1605 il y avait un monde sans Cervantes et sans Don Quichotte, et des gens vivaient à cette époque dont personne ne se souvient. Alors, il y a un pouvoir de mémoire durable dans le fait littéraire qui doit beaucoup influencer notre travail et dépasser nos personnes. C'est l'humilité de dire: l'œuvre est ouverte, et on la communique à un lecteur parce qu'on est écrivain. Je suis aussi lecteur, bien sûr, et j'ai aussi ce sentiment que $T i$ rano Banderas, de Valle-Inclán, ou n'importe quel roman de Balzac me sont ouverts, comme lecteur d'aujourd'hui.

C'est en cela que consistel'échange : l'écrivain doit laisser le roman ouvert pour les futurs lecteurs, mais l'écrivain en tant que lecteur doit être ouvert à l'œuvre qui précède la sienne.

L'intertextualité littéraire est-elle le moteur de la genèse de plusieurs de vos récits?

Si vous le dites, c'est sûrement vrai! Je ne suis pas le seul écrivain à le faire. Pourquoi? Parce qu'une œuvre littéraire hérite d'autres ouvres, et ajoute quelque chose qui auparavant n'y était pas, et dans dix ou vingt ans quelqu'un parlera de la nôtre. Je reviens d'Aix-en-Provence où j'ai rencontré beaucoup de jeunes écrivains chiliens, colombiens, mexicains, qui sont deux fois plus jeunes que moi et qui font ce que je n'aurais pas pu imaginer il y a quarante ans. Mais ils le font parce que García Márquez, Mario Vargas Llosa, Cortázar, Carpentier, Borges et Asturias et moi avons écrit auparavant, et qu'eux écrivent par assimilation; ils écrivent de nouveaux livres, des livres insoupçonnés, tout comme moi j'étais insoupçonné pour Miguel Angel Asturias.

Chaque œuvre crée quelque chose de nouveau, de nouvelles perspectives, et signale la dette qu'elle a envers la tradition, mais aussi, en tant que création, la dette qu'elle a envers l'avenir.

De vos débuts dans l'écriture à aujourd'hui, le rôle du romancier a-til changé? Quelles sont les difficultés auxquelles se heurte l'écrivain à notre époque?

Je crois que c'est la vision du romancier, non son rôle, qui a changé.

Je n'aurais pas deviné ce qui est arrivé en Afrique du Nord, alors que Barack Obama l'avait annoncé au Caire. Des choses historiques nouvelles qu'on n'aurait pas même imaginées adviennent dans le monde: soudain l'Égypte a changé, et Mohammed Moubarak, et le dictateur de Tunisie, et Mohammed Khadafi sont partis : que s'est-il passé? Pourquoi est-ce arrivé? Pourquoi un homme politique peut-il le prévoir, et pas un écrivain, alors que nous sommes censés être les maîtres de l'imagination?

Ainsi, pour répondre à votre question, on ne peut pas établir de catégories fixes, peu flexibles. Le travail littéraire change en permanence, 
nous vivons dans un monde chaque jour plus mobile et plus difficile à cause de la concurrence. Les révolutions d'Afrique du Nord se sont faites à partir de Facebook, de Twitter, de nouveautés qui n'existaient pas il y a seulement dix ans. Des changements aussi gigantesques et soudains posent un problème à l'écrivain : quelle valeur a l'imagination pour continuer d'exister dans un monde de changement perpétuel? Qu'allons-nous dire? Qu'allons-nous imaginer? Je crois qu'aujourd'hui le danger est plus grand que jamais car les médias sont très accessibles, très rapides et en constante transformation.

Mais je crois à l'existence d'une imagination proprement littéraire qui transcende les changements de la communication quotidienne, sinon on n'écrirait pas. Je sens qu'il en a toujours été ainsi : l'essor de la presse quotidienne après la Révolution Française n'a pas empêché Balzac et Flaubert d'écrire, pas plus que le succès du cinéma n'a arrêté John Dos Passos et Faulkner. Le développement des moyens de communication actuel ne va pas nous empêcher d'écrire non plus. C'est-à-dire qu'il y a une vision inhérente au fait littéraire qui transcende d'autres moyens de communication, de quelque époque que ce soit.

Comment préserver la vertu littéraire qui consiste à écrire des livres à partir de l'imagination et de la mémoire va être, ou plutôt a toujours été, notre défi. C'est un défi que de coexister avec toute la nouveauté du monde.

\section{Le mythe littéraire peut-il garder} son pouvoir aujourd'hui dans le domaine politique ou social?

Toutes les idéologies sont des mythes et s'écroulent facilement. Quand on pense à l'idéologie du national-socialisme allemand, "se lo llevó la chingada " comme on dit au Mexique, elle est partie en fumée, franchement elle a disparu. Les œuvres d'art durent plus longtemps que les idéologies politiques, sans nul doute; c'est là une bonne raison de continuer à travailler.

Le roman a-t-il un rôle central face à ce fait social qu'est l'augmentation du trafic de drogue au Mexique et en Amérique Centrale?

La littérature n'a qu'un rôle: celui d'exercer l'imagination et la mémoire face à n'importe quel fait. J'ai écrit un roman sur le narcotrafic, Adán en Edén, en me disant que je ne voulais pas écrire ce que j'écrirais dans un journal comme La Reforma ou El País pour dénoncer le narcotrafic, que je devais le tourner autrement, par l'imagination. C'est un roman comique sur un fait tragique, mais c'est ce qui pour moi, en tant qu'écrivain, fait sa nouveauté. La littérature est bien plus complexe, elle exige davantage, il faut transformer ce que nous voyons tous les jours en quelque chose qui a un autre sens, indépendant de la réalité, mais qui finit par en faire partie. 
Dans le champ littéraire, vous considérez-vous comme une sorte de moraliste ou d'immoraliste?

Je me considère comme une sorte d'immoraliste depuis que j'ai lu le livre d'André Gide L'immoraliste. Il se passe quelque chose de curieux dans la littérature : c'est très difficile d'écrire sur des gens bons. Miguel de Cervantes l'a fait avec Don Quichotte, Dickens avec Pickwick, Dostoïevski dans L'Idiot. Mais il est beaucoup plus facile de parler des méchants, c'est ce qui donne de l'imagination et de la force. C'est pour cela que le Quichotte est un chef-d'œuvre, parce qu'il parvient à consacrer la bonté du personnage et de la réalité qu'il vit, mais, hormis cet exemple, c'est très difficile. En fait, les bons écrivains sont tous immoraux!

Quels sont les défis principaux de la littérature latino-américaine du $\mathrm{XXI}^{\mathrm{e}}$ siècle?

D'être comprise partout. Certains romans du passé n'étaient compris qu'en Bolivie, en Équateur ou en Colombie. Aujourd'hui il faut être capable de communiquer, d'être compris par un lecteur dit "global», je dirais plutôt " international ».

Si Cent ans de solitude est traduit dans presque cinquante langues, cela signifie que ce n'est pas seulement un roman colombien mais un roman ukrainien, tibétain, que sais-je encore, un roman que l'on comprend partout. Aussi le bon écrivain sait-il tirer grand profit de ses racines locales, comme Faulkner : qui est plus localiste que Faulkner parlant du Sud des États-Unis? Pourtant ses romans sont compris dans n'importe quelle langue! C'est cela le secret de la bonne littérature. 